\title{
NONCONTACT TRUE TEMPERATURE MEASUREMENT ॥
}

\author{
MARK C. LEE AND JAMES L. ALLEN
}

Jet Propulsion Laboratory, California Institute of Technology, Pasadena, CA 91109

\section{ABSTRACT}

A laser pyrometer has been developed for acquiring the true temperature of a levitated sample. The reflectivity is measured by first expanding the laser beam to cover the entire cross-sectional surface of the diffusive target. The reflectivity calibration of this system is determined from the surface emissivity of a target with a blackbody cavity. The emissivity of the real target can then be calculated. The overall system constant is obtained by passively measuring the radiance of the blackbody cavity (emissivity $=1.0$ ) at a known, arbitrary temperature. Since the photo sensor used is highly linear over the entire operating temperature range, the true temperature of the target can then be computed. The latest results available from this on-going research indicate that true temperatures thus obtained are in very good quantitative agreement with thermocouple measured temperatures.

\section{INTRODUCTION}

Many nonintrusive thermophysical measurements require an accurate determination of the true temperature of the sample. For example, in space investigations of the undercooling behaviors of bulk metals, alloys, and refractories, it is mandatory that the measurements be made in a noncontact manner to prevent premature nucleation and crystallization. Unfortunately, conventional remote sensing techniques just detect the radiation of the sample and thus only the 'brightness temperature'; whenever the two sample surfaces show the same magnitude of radiance in the prescribed frequency band, the temperatures of the two samples are considered equal regardless of the emissivity characteristics of the 
individual samples. In an improved version of this approach, an estimated single value of the emissivity for the entire temperature region of interest can be 'dialed in' in an effort to reduce the uncertainty introduced by the totally unknown emissivity. Still another approach employs a shorter-wavelength bandwidth for the photo detector to decrease the sensitivity of the calculated temperature to the emissivity [1]. However, without the in-situ real time emissivity information, it is not possible to accurately determine the true temperature of the sample.

This paper will report the latest data available from on-going research to develop a noncontact true temperature measurement technique. In addition to the conventional passive measurement of the radiation power, an active laser beam is incorporated for acquiring the reflectivity of the sample surface. The emissivity and thus the true temperature of the sample can be derived from the measured reflectivity and radiance signals.

Results previously reported showed excellent correlations between true temperatures acquired by this technique and those by thermocouple [2]. In these initial results, the pyrometer and thermocouple temperatures were normalized at one point using a different system constant for each target material. The data reported here represent a marked improvement over this initial data, with the need to normalize the data and use material dependent system constants having been eliminated. Although further refinement is needed to produce a viable technique, these latest results have reached the point of very good quantitative agreement between pyrometer and thermocouple measured temperatures.

\section{THEORETICAL BACKGROUND}

The uncertainty in a temperature measurement can be expressed as:

$$
\Delta T / T=\left[(\Delta \varepsilon / \varepsilon)^{2}+(\Delta I / /)^{2}\right]^{1 / 2} \lambda \kappa T / h c
$$

where $\Delta T$ is the uncertainty in measuring the true absolute temperature $T, \varepsilon$ is the spectral emissivity, $I$ is the spectral radiation intensity at wavelength $\lambda_{1} c$ is the speed of light, and $\mathrm{k}$ and $\mathrm{h}$ are the Boltzmann and Planck constants, respectively. For practical application in pyrometry, I can be closely approximated by Wien's equation: 


$$
I(\lambda, T)=c_{1} \varepsilon(\lambda, T) \lambda^{-5} \exp \left(-c_{2} / \Lambda T\right)
$$

where $c_{1}$ and $c_{2}$ are constants with $c_{1}$ being $3.7403 \times 10^{-4}$ watt $\cdot \mu \mathrm{m}^{2}$ and $c_{2}$ being $1.4384 \times 10^{4} \mu \mathrm{m}^{\circ} \mathrm{K}$. Therefore, $\mathrm{I}(\mathrm{\Lambda}, \mathrm{T})$ is in watts $/ \mathrm{m}^{2} \cdot \mu \mathrm{m}$.

Without knowing the detailed behavior of $\varepsilon$ as a function of $\lambda$ and $T$, shorter wavelengths are traditionally chosen so that $\mathrm{I}$ is more sensitive to the exponential term in equation (2). This manipulation at least reduces the relative contribution of $\Delta \varepsilon$ to the overall $\Delta \mathrm{T}$. In addition, by assuming $\varepsilon$ is constant over the wavelength and temperature range of interest, the quotients of two or more I's can be taken to eliminate $\varepsilon$ from consideration completely. The theoretical limitations of these so called 'multiple color pyrometry' approaches and their derivatives were reviewed in depth recently by Coates and Nordine $[3,4]$. Two conclusions indicated by these studies are that 1) the single color pyrometer provides the least experimental error in true temperature measurement, and 2) further improvements are needed to provide true temperature data in order to meet more stringent requirements in the next generation of thermophysical measurements.

In this paper a 'laser pyrometer' technique will be presented for simultaneous measurements of emissivity and radiation. In this technique, the surface emissivity, $\varepsilon_{s}$, of a calibration target with a blackbody cavity can be obtained by using the radiance channel of the pyrometer to alternately measure the radiance signal of the calibration target surface, $\mathrm{V}_{\mathrm{rs}}$, and the radiance signal of the blackbody cavity, $\mathrm{V}_{\mathrm{rb}}$. By the definition of emissivity:

$$
\varepsilon_{s}=V_{r s} / V_{r b}
$$

The reflectivity, $\Gamma_{\mathrm{s}}$, of the calibration target surface is given by :

$$
\Gamma_{s}=V_{e s} / V_{\ell c}
$$

where $\mathrm{V}_{\ell s}$ is the laser (reflectivity) channel signal of the calibration target surface and $V_{\ell c}$ is the laser channel calibration factor. By Kirkhoff's law, $\varepsilon_{S}=1-\Gamma_{S}$, and the calibration factor, $V_{\ell c}$, can then be found:

$$
V_{\ell c}=V_{\ell s} /\left(1-\varepsilon_{s}\right)
$$

Once $V_{\ell c}$ is known, the spectral emissivity of a real target, $\varepsilon_{t}$, can then be calculated: 


$$
\varepsilon_{t}=1-V_{e t} / V_{\ell c}
$$

where $\mathrm{V}_{\ell t}$ is the measured laser (reflectivity) signal of the real target.

A conventional passive radiance measurement yields the radiance signal of the target, $V_{r t}=a_{1} I(\Lambda, T)$. From equation (2),

$$
T\left({ }^{\circ} K\right)=15916 /\left\{\ln \left[\varepsilon(\lambda, T) / C_{s y s} V_{r t}\right]\right\}
$$

where $\mathrm{C}_{\mathrm{sys}}$, the system constant, can be obtained by calibrating the laser pyrometer against the blackbody cavity of the calibration target at a known, arbitrary temperature in the following manner.

The system constant can be calculated by solving equation (7) for $\mathrm{C}_{\mathrm{sys}}$ and substituting the appropriate parameters as measured using the blackbody calibration target. This can be done in two ways:

$$
C_{s y s}=\varepsilon_{s} / V_{r s} \exp (C / T)
$$

or

$$
C_{s y s}=\varepsilon_{b} / V_{r b} \exp (C / T) .
$$

$\varepsilon_{\mathrm{s}}$ is the emissivity of the calibration target surface, and $\varepsilon_{\mathrm{b}}=1$ is the assumed emissivity of the blackbody cavity. $\mathrm{V}_{\mathrm{rs}}$ and $\mathrm{V}_{\mathrm{rb}}$, are the radiance signals of the calibration target surface and blackbody cavity, respectively. $\mathrm{C}$ is the constant 15916, and $\mathrm{T}$ is the true temperature as determined by thermocouple. A comparison of these two values of the system constant will provide a measure of the validity of this approach.

\section{EXPERIMENTAL APPROACH}

The prototype laser pyrometer developed consists of a passive radiance channel and an active laser channel, respectively. For all targets, chromel-alumel thermocouples were placed in intimate thermal contact at appropriate positions on the surfaces of the samples. This type of thermocouple places an upper limit on the calibration to not exceed approximately $1100^{\circ} \mathrm{C}$ at the present time. Each sample 
was then heated by an electromagnetic coil powered by a Lepel 60kW RF generator. The RF coil is enclosed in a stainless steel belljar which can be evacuated to a diffusion pump vacuum.

Originally, shielded thermocouples were used to minimize possible RF effects while monitoring the target temperatures. Further tests indicated that exposing the thermocouple junction and placing it in direct contact with the sample surface produced more noise, but provided less of a heat sink, and gave a more accurate measurement of the target surface temperature than using shielded thermocouples.

The pyrometer views all targets through an optical window made of quartz which is transparent to $.904 \mu \mathrm{m}$ laser infrared. The laser is pulsed at $2000 \mathrm{pps}$ with a time constant of 200ns. Depending on applications, the data acquisition rate can be as fast as $5 \times 10^{6}$ data per second. However, in this initial calibration phase, each datum point represents an integration of $50 \mathrm{~ms}$ or 100 returned pulses. The radiance signal is also integrated for $10 \mathrm{~ms}$ before digitization. The radiance and reflectivity signals are sampled sequentially, and each data pair is sent at one second intervals to a PDP $11 / 23$ computer for further processing.

For a typical calibration run, the belljar is first purged with high purity argon gas and evacuated to mechanical pump vacuum. The procedure is repeated twice before the belljar is backfilled with 10 psia argon gas. The sample is then heated up by incrementally increasing the RF power. Once the target temperature as monitored by the thermocouple has stabilized at each increment, the pyrometer is then refocused to maximize the reflectivity signal. When the pyrometer is properly focused, fifty data points are taken by the computer and averaged. Each processed reflectivity datum point, therefore, represents an average of 5,000 returned raw laser pulses. The schematic for this laser pyrometer calibration system is shown in figure 1.

Originally the laser channel was calibrated by measuring the reflected laser signal from a gold-coated sphere (reflectivity $=0.99$ ). In order to improve upon previous results, a method had to be established that 1) eliminated the need for a gold-coated target whose reflectivity must be independently verified, and 2) provided the laser channel calibration and system constant using the same experimental apparatus and optical paths as would be used with the real targets.

Therefore, to permit a calibration procedure as described in the previous section, two types of targets were made: 1 ) a blackbody calibration target to determine $V_{\ell_{s}}$ and $\mathrm{C}_{\mathrm{sys}}$, and 2) spherical targets of different materials to allow the comparison of 
pyrometer and thermocouple temperatures. A cylindrical calibration target was made from stainless steel with a blackbody cavity in the center. The diameter of the cavity opening is the same as the diameter of the spherical targets $(0.95 \mathrm{~cm})$, and the outside diameter allows more than enough room for the pyrometer to view the surface without overlapping the outer edge or the cavity edge at the center. The pyrometer can be moved to alternate the point of focus between the surface and the cavity. All surfaces have been throughly sand-blasted to make all pyrometer viewing areas as diffuse as possible.

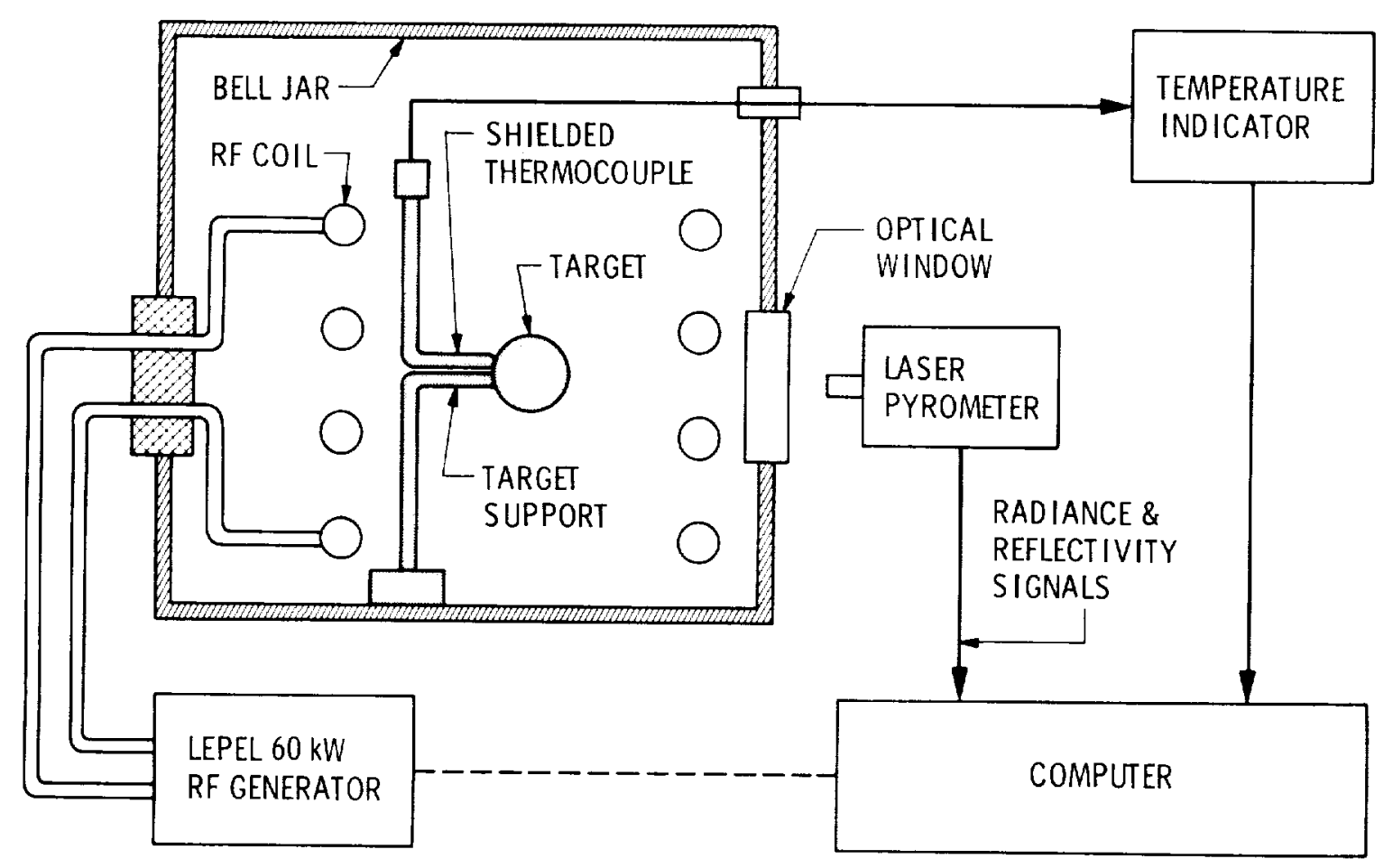

Figure 1. Laser pyrometer calibration system schematic.

The calibration target is placed in the coil at the same position relative to the quartz window and pyrometer using the same atmosphere and pressures as would be used for the spherical targets. The calibration target was initially oxidized in order to turn on the radiance channel of the pyrometer at about $750^{\circ} \mathrm{C}$. Therefore, to follow the procedure as described in the previous section, the laser pyrometer 
monitors three parameters of the blackbody calibration target: the radiance signal of the surface, $V_{r s}$, the laser (reflectivity) signal of the surface, $V_{\ell s}$, and the radiance signal of the blackbody cavity, $V_{r b}$. A schematic of the calibration target, the pyrometer and the pertinent calibration parameters is shown in figure 2 .

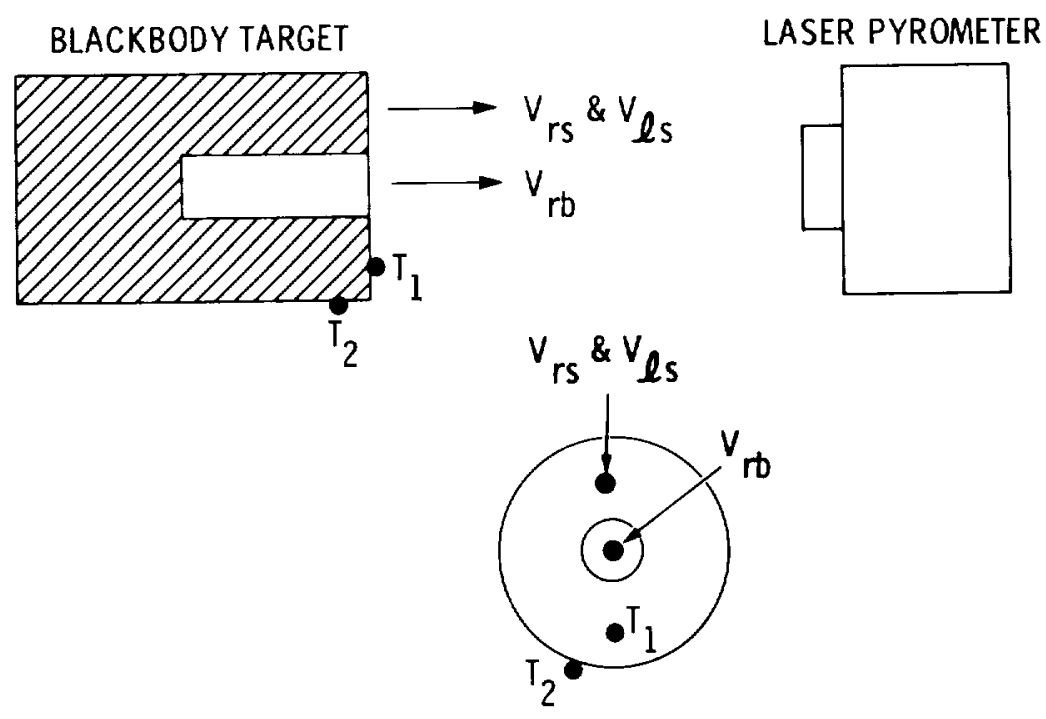

Figure 2. Calibration target schematic and parameters.

The calibration target has a high thermal mass to minimize errors due to temperature gradients on the surface. Two chromel-alumel thermocouples are placed at locations $T_{1}$ and $T_{2}$ to monitor the temperature of the calibration target as it is heated in the RF coil. $T_{1}$ is located at the same radius but opposite the focal point where the pyrometer monitors the calibration target surface. $\mathrm{T}_{2}$ allows any gradients between the outer edge and the front surface to be monitored.

Once $\mathrm{V}_{\ell c}$ and $\mathrm{C}_{\mathrm{sys}}$ have been determined from the blackbody calibration, the true temperatures of the spherical targets can be measured. Three target materials were used: stainless steel, titanium, and carbon. Because the spectral emissivity can depend strongly on the viewing angle of the spherical surface, the two metal targets were thoroughly sand-blasted to make the surfaces as diffuse as possible. Also, the two metal targets were oxidized to activate the radiance channel at around $750^{\circ} \mathrm{C}$. 


\section{RESULTS AND DISCUSSION}

Figures 3 and 4 show the reflectivity calibration factor, $V_{\ell c}$, and the system constant, $\mathrm{C}_{\mathrm{sys}}$, respectively, as functions of temperature as obtained from the blackbody calibration target. The temperatures were obtained from the chromelalumel thermocouple located at $\mathrm{T}_{1}$. For the ideal pyrometer system, it is most desirable that both $\mathrm{V}_{\ell_{c}}$ and $\mathrm{C}_{\mathrm{sys}}$ be independent of temperature and material. In both cases, $\mathrm{V}_{\ell c}$ and $\mathrm{C}_{\mathrm{sys}}$ are both clearly temperature dependent. Even though $\mathrm{V}_{\ell \mathrm{c}}$ and $\mathrm{C}_{\mathrm{sys}}$ vary with temperature, the average values of each were used over the entire temperature range for each spherical target. By forcing $\mathrm{V}_{\ell \mathrm{c}}$ and $\mathrm{C}_{\mathrm{sys}}$ to be constants, a comparison of the current data to the ideal can be made. Further investigation is needed to determine if this dependence can be decreased or eliminated.

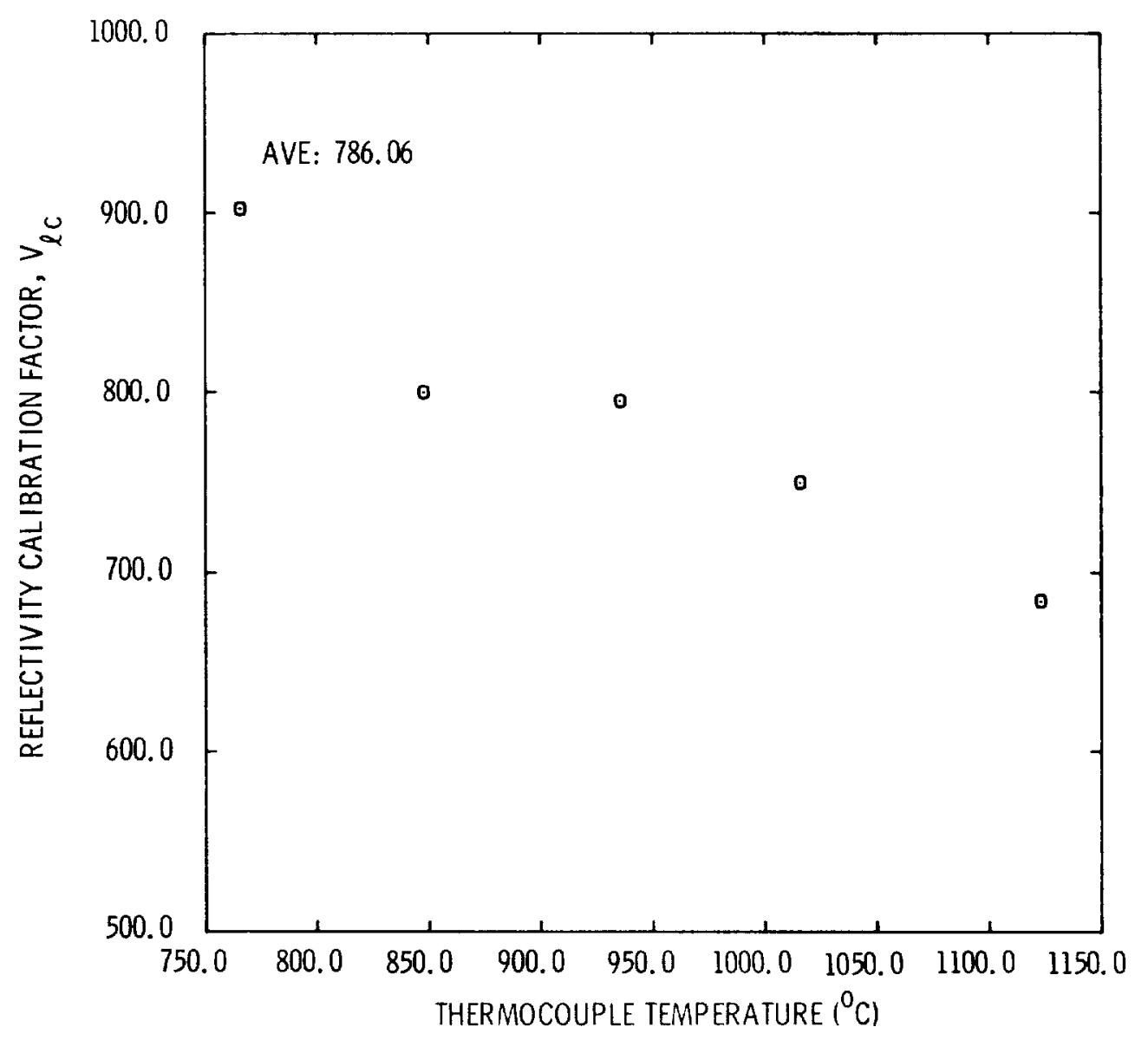

Figure 3. Reflectivity calibration factor, $V_{\ell c}$, as a function of thermocouple temperature. 


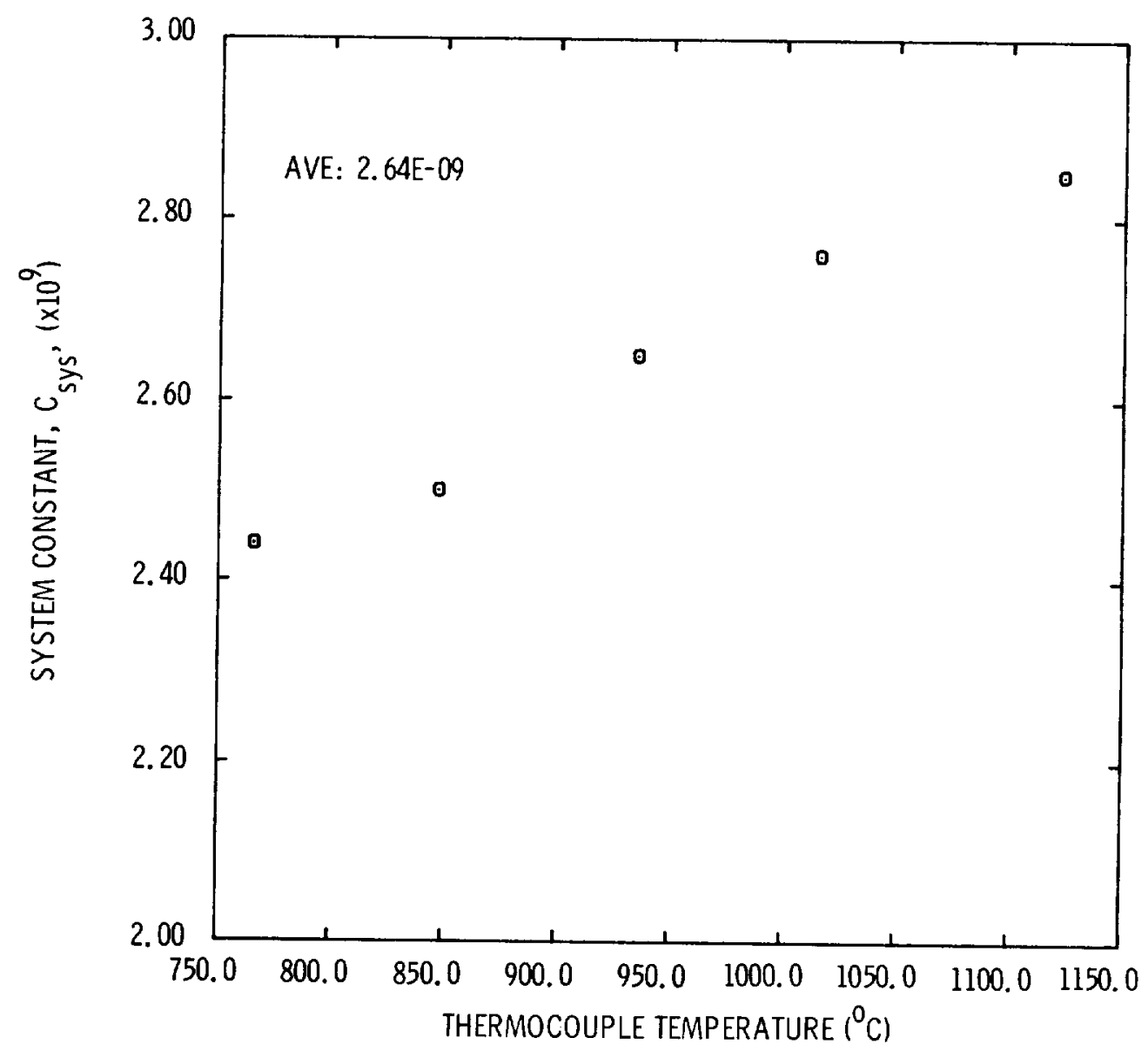

Figure 4. System constant, $\mathrm{C}_{\mathrm{sys}}$, versus thermocouple temperature.

$V_{\ell c}$ varied between 684.0 and 902.2 yielding an average of $786.06 . \mathrm{C}_{\mathrm{sys}}$ varied between $2.86 \times 10^{9}$ and $2.43 \times 10^{9}$ yielding an average of $2.64 \times 10^{9} . \mathrm{C}_{\text {sys }}$ was calculated using both equations ( 8 ) and (9) with the discrepancy between the individual data points being $1.2 \%$ in the worst case and averaging less than $0.4 \%$. This kind of agreement indicates that the approach taken for calibration is quite valid. 
The raw data from the passive radiance channel for all three targets are shown in figure 5. In general they closely follow a curve with increasing slope at higher temperatures.

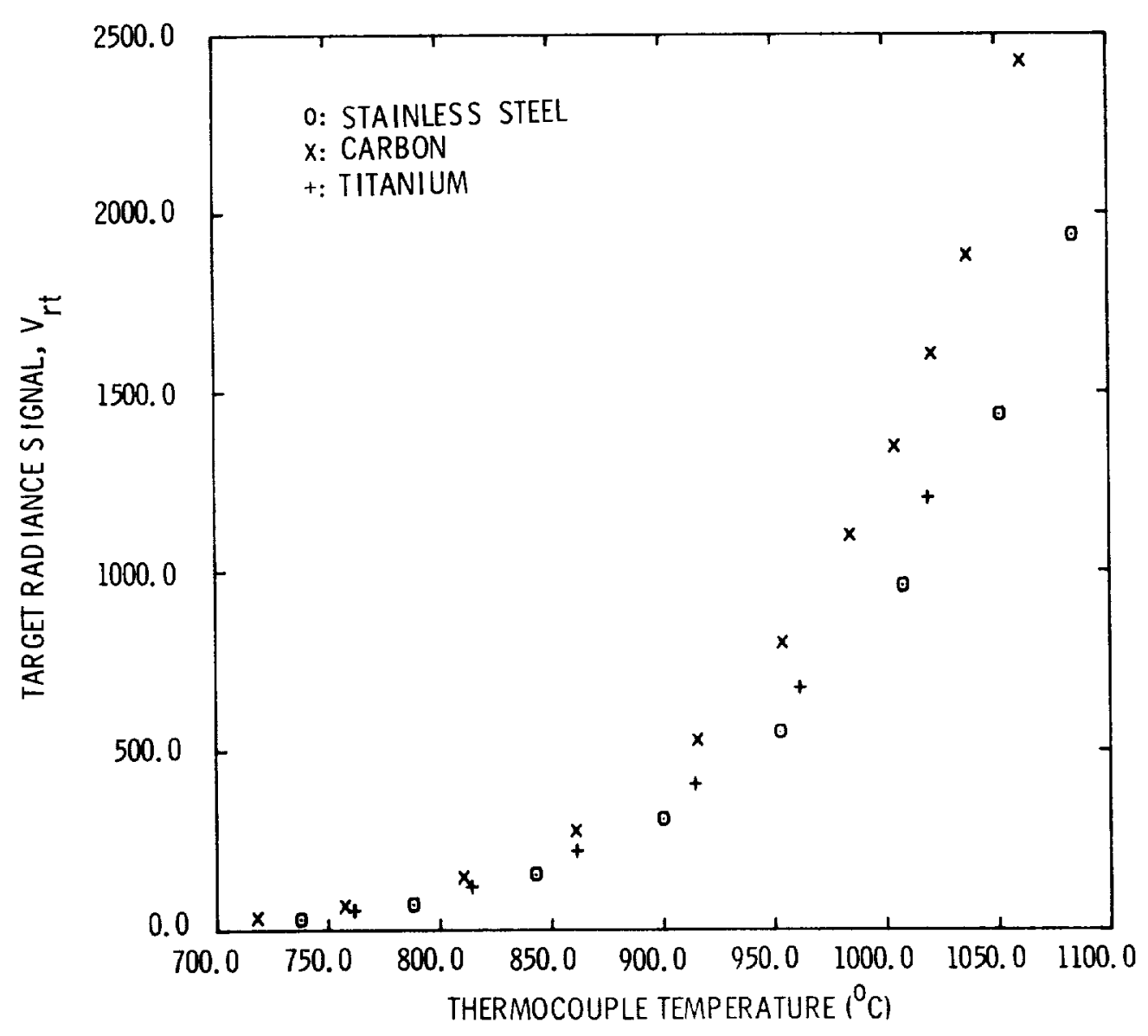

Figure 5. Raw radiance data versus thermocouple temperature.

Using the average reflectivity calibration factor, $\mathrm{V}_{\ell c}=786.06$, and the raw reflectivity data for each sample, the emissivity for each target can then be calculated using equation (6). The data for all three targets are plotted in figure 6 . Note that the emissivity of the stainless steel target increased with temperature. 
This process was completely reversible with the emissivity decreasing as the target cooled down. This is completely expected since the emissivity of iron oxide increases with temperature. The emissivity of carbon decreases with increasing temperature. which is just the opposite of what was observed. The carbon became more and more pitted as the temperature increased probably due to residual oxygen in the target or the atmosphere. The increase in the carbon emissivity was non-reversible and remained at the higher level after the target had cooled down. It is important to note, however, that any temperature variation of the emissivity is irrelevant in this technique since it is measured in-situ and in real time.

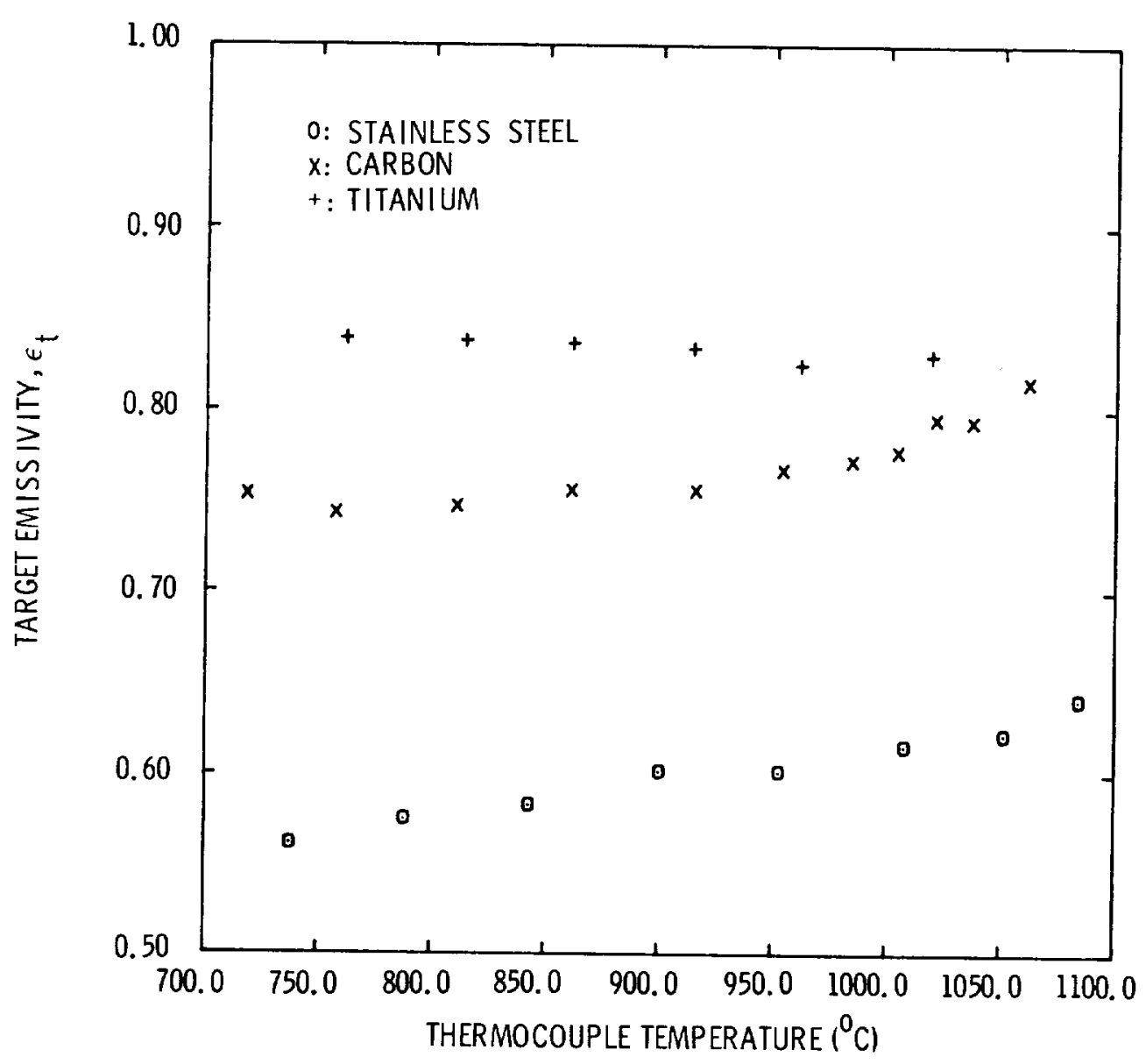

Figure 6. Target emissivity, $\varepsilon_{t}$, as a function of thermocouple temperature. 
Using the average system constant, $\mathrm{C}_{\text {sys }}=2.64 \times 10^{9}$, for all targets, pyrometer temperatures can be plotted against thermocouple temperatures, as shown in figure 7 . The $45^{\circ}$ line represents the one-to-one correspondence between the temperatures. The pyrometer temperatures for the carbon are consistently too high while those for titanium are consistently too low. The stainless steel pyrometer temperatures fall almost on the line. The fact that the blackbody calibration target was also made of stainless steel suggests that the system constant has a slight material dependence.

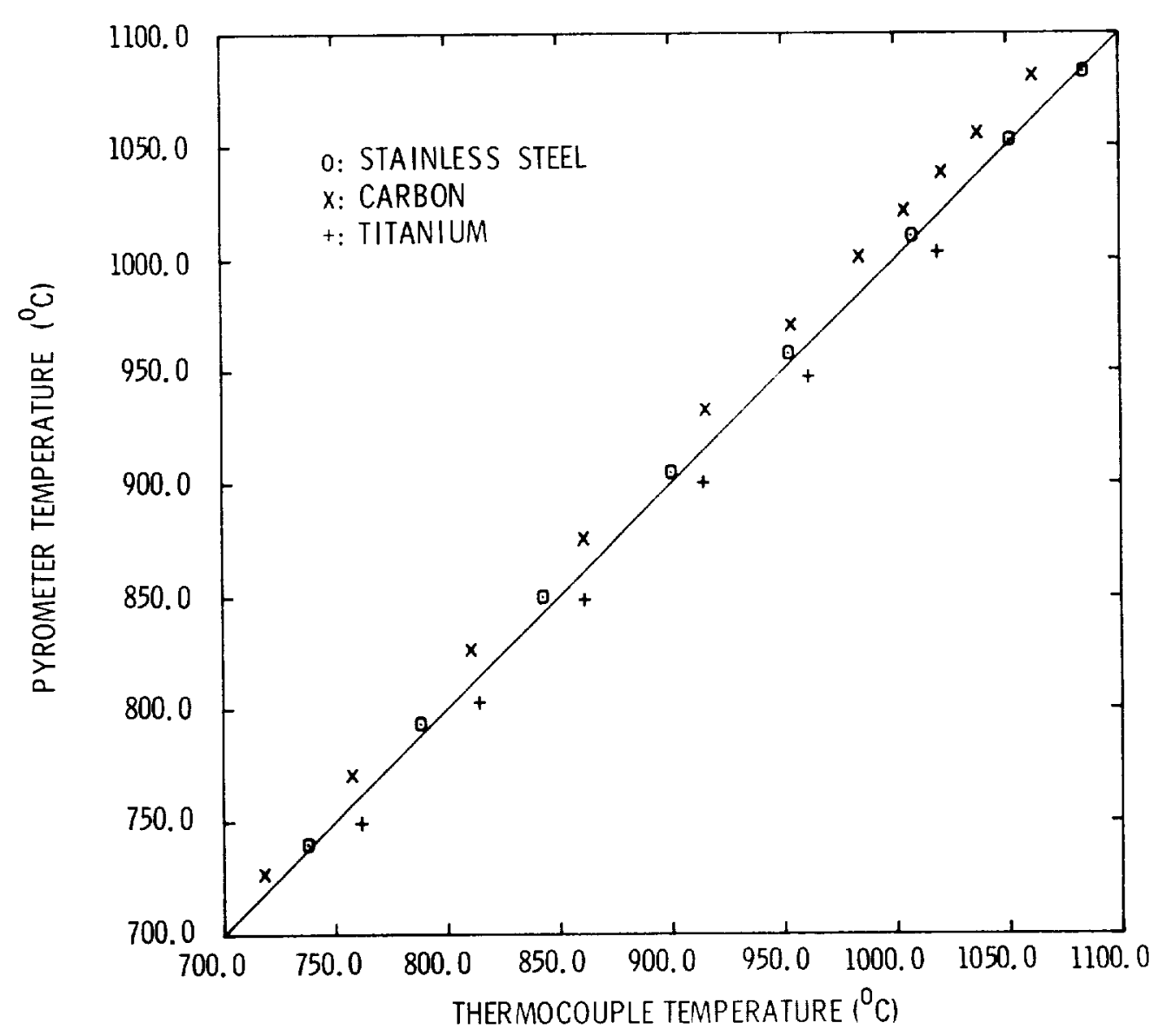

Figure 7. Pyrometer temperature versus thermocouple temperature. 
However, these deviations are not very large. The carbon target produced the largest errors, with the worst $\Delta \mathrm{T}$ being $19.5^{\circ} \mathrm{C}$. The worst discrepancies for all three targets were $1.8 \%$ for carbon, $-1.6 \%$ for titanium, and $0.87 \%$ for stainless steel. These values are approaching the accuracies with which the thermocouple measurements can be made. For example, the thermocouple lead wires provide a heat sink for the target and the thermocouple junction. This was evident for all targets from the fact that the lead wires remained black even when the junction and target were glowing very brightly. The thermocouple junctions were spot welded to the stainless steel and titanium targets, which was not possible with the carbon target. Thus, the thermocouple junction on the carbon target could very easily have been at a slightly lower temperature than the target itself. Additionally, the titanium target reacted quite strongly with the thermocouple junction and the argon atmosphere which could also affect the thermocouple readings.

Two assumptions were also made regarding the blackbody target calibration. First of all, it was assumed that no temperature gradient existed on the front surface of the calibration target between $\mathrm{T}_{1}$ and the pyrometer view point. Secondly, the assumption was made that it was valid to extend the calibrations using the flat surface of the calibration target to the curved surfaces of the spherical targets. This assumption is only valid if all surfaces were perfectly diffusive.

Also, average values of the reflectivity calibration factor and the system constant were used for the entire temperature range even though both exhibited definite temperature dependences. The final results indicate that the pyrometer temperatures are insensitive to temperature variations in both $V_{\ell c}$ and $\mathrm{C}_{\mathrm{sys}}$ which is exactly what is desired. Therefore, it is not obvious that any temperature or material dependences exist in either parameter. In light of the error contributing factors mentioned above, it is very promising that the discrepancies between the thermocouple and pyrometer temperatures are less than $2 \%$.

\section{CONCLUSIONS}

A noncontact true temperature measurement technique has been presented, which employs the principle of simultaneous measurement of the radiance and reflectivity of the target sample. Current data represent a definite improvement over previously reported results and indicate that the material dependence of the 
system constant has been greatly reduced with overall discrepancies in temperatures of less than $2 \%$. The progress made thus far indicates that further refinement of this laser pyrometry technique will provide an improvement in accuracy of at least an order of magnitude as compared to passive techniques.

\section{ACKNOWLEDGEMENTS}

The authors are indebted to Dr. A. Stein of Quantum Logic Corporation for stimulating discussions. The authors are also grateful to Quantum Logic for modifying their existing commercial pyrometer (Model No. 1300C) to meet our specific requirements. Thanks are also due to $\mathrm{Mr}$. G. Ladner, Jr. for technical assistance. This work represents one phase of research carried out at the Jet Propulsion Laboratory, California Institute of Technology, under contract with the Microgravity Science and Applications Division, the National Aeronautics and Space Adminstration.

\section{REFERENCES}

1. M. C. Lee and J. L. Allen, Materials Research Society Symposia Proceedings, $\underline{87}$, 285 (1987).

2. M. B. Frish, M. N. Spencer, N. E. Wolk, J. S. Werner, and H. A. Miranda, Jr., Physical Sciences Inc. Final Report to Jet Propulsion Laboratory/NASA titlted, "Multi-Color Pyrometer for Materials Processing in Space" , July 15, 1986.

3. P. B. Coates, Metrologia 17, 103 (1981).

4. P. C. Nordine, High Temp. Sci., 21, 97 (1986). 